alizes the theorem of Thompson and Tait. We can prove, in fact, that a condition for an affirmative answer to our question is that, on any tube of $(S)$, either all or none of the transversal curves should be closed.

Trinity College, Dublin, IRELAND

\title{
ON THE CONDITION THAT TWO ZEHFUSS MATRICES BE EQUAL
}

\author{
BY D. E. RUTHERFORD
}

1. Introduction. In a recent paper* Williamson has considered matrices whose sth compounds are equal. The present paper considers the somewhat analogous problem of finding the conditions that two Zehfuss matrices be equal.

Suppose that $R$ is a matrix of $n_{1}$ rows and $m_{1}$ columns whose $i j$ th element is $r_{i j}$, and that $P$ is another matrix of $n_{2}$ rows and $m_{2}$ columns. Now, if the matrix $Q$ of $n_{1} n_{2}$ rows and $m_{1} m_{2}$ columns can be partitioned into submatrices each of $n_{2}$ rows and $m_{2}$ columns such that the $i j$ th submatrix is $r_{i j} P$, then $Q$ is a $Z e h f u s s$ matrix $\dagger$ or the direct product matrix $\ddagger$ of $R$ and $P$. We shall write

$$
Q=R\langle P\rangle=\langle P\rangle R \text {. }
$$

In general, however, $R\langle P\rangle \neq\langle P\rangle R$.

It is the purpose of this paper to find out under what conditions the matrix equation

$$
A\langle B\rangle=C\langle D\rangle
$$

is true. That is, we shall find the most general form of the matrices $A, B, C, D$ when the above equation holds.

2. The Simplest Case. We shall begin by considering the simplest case, where $A, B, C, D$ are row vectors, where $A$ and $D$ are of order $m_{1}$, where $B$ and $C$ are of order $m_{2}$, and where

$$
\left(m_{1}, m_{2}\right)=1 \text {; }
$$

that is to say, $m_{1}$ and $m_{2}$ are prime to one another. Suppose that

* J. Williamson, this Bulletin, vol. 39 (1933), p. 109.

$\dagger$ G. Zehfuss, Zeitschrift für Mathematik und Physik, vol. 3 (1858), p. 298.

$\ddagger$ L. E. Dickson, Algebras and Their Arithmetics, p. 119. 


$$
\begin{aligned}
A & =\left[a_{1}, a_{2}, \cdots, a_{m_{1}}\right], \quad B=\left[b_{1}, b_{2}, \cdots, b_{m_{2}}\right], \\
C & =\left[c_{1}, c_{2}, \cdots, c_{m_{2}}\right], \quad D=\left[d_{1}, d_{2}, \cdots, d_{m_{1}}\right],
\end{aligned}
$$

and let $m_{2}>m_{1}$. From definition, we have $A\langle B\rangle=\left[a_{1} b_{1}, a_{1} b_{2}, \cdots, a_{1} b_{m_{2}}, a_{2} b_{1}, \cdots\right.$, and $\left.a_{2} b_{m_{2}}, \cdots, a_{m_{1}} b_{1}, \cdots, a_{m_{2}} b_{m_{2}}\right]$

$$
\begin{array}{r}
C\langle D\rangle=\left[c_{1} d_{1}, c_{1} d_{2}, \cdots, c_{1} d_{m_{1}}, c_{2} d_{1}, \cdots,\right. \\
\left.c_{2} d_{m_{1}}, \cdots, c_{m_{2}} d_{1}, \cdots, c_{m_{2}} d_{m_{1}}\right] .
\end{array}
$$

Identifying these two row vectors element by element, we get $m_{1} m_{2}$ equations, determining the relations which must hold between the elements of $A, B, C, D$. Amongst these $m_{1} m_{2}$ equations, consider the following:

$$
\begin{aligned}
& \alpha_{1} b_{1}=\gamma_{1} d_{1}, \\
& \alpha_{2} b_{2}=\gamma_{2} d_{1} \text {, } \\
& \alpha_{m_{2}-m_{1}+1} b_{m_{2}-m_{1}+1}=\gamma_{m_{2}-m_{1}+1} d_{1},
\end{aligned}
$$

where each $\alpha$ represents some one of $a_{1}, \cdots, a_{m_{1}}$, and each $\gamma$ some one of $c_{1}, \cdots, c_{m_{2}}$. A little consideration will show that no two $\alpha$ 's represent the same $a$ and no two $\gamma$ 's represent the same $c$. It is obvious that $\alpha_{1}=a_{1}$ and $\gamma_{1}=c_{1}$. From equations (1) and from the construction of $A\langle B\rangle$ and $C\langle D\rangle$ it follows that

$$
\begin{aligned}
& \alpha_{1}\left[b_{1}, \cdots, b_{m_{1}}\right]=\gamma_{1}\left[d_{1}, \cdots, d_{m_{1}}\right], \\
& \alpha_{2}\left[b_{2}, \cdots, b_{m_{1}+1}\right]=\gamma_{2}\left[d_{1}, \cdots, d_{m_{1}}\right] \text {, } \\
& \alpha_{m_{2}-m_{1}+1}\left[b_{m_{2}-m_{1}+1}, \cdots, b_{m_{2}}\right]=\gamma_{m_{2}-m_{1}+1}\left[d_{1}, \cdots, d_{m_{1}}\right] .
\end{aligned}
$$

From equations (2) we deduce

and

$$
b_{2}=\frac{\gamma_{1}}{\alpha_{1}} d_{2}=\frac{\gamma_{2}}{\alpha_{2}} d_{1}, \text { whence } \frac{d_{2}}{d_{1}}=\frac{\gamma_{2} \alpha_{1}}{\gamma_{1} \alpha_{2}}=s, \text { say, }
$$

$$
b_{3}=\frac{\gamma_{2}}{\alpha_{2}} d_{2}=\frac{\gamma_{3}}{\alpha_{3}} d_{1}, \text { whence } \frac{d_{2}}{d_{1}}=\frac{\gamma_{3} \alpha_{2}}{\gamma_{2} \alpha_{3}}=s,
$$

and so on. In this way we can show that 


$$
s=\frac{\gamma_{2} \alpha_{1}}{\gamma_{1} \alpha_{2}}=\frac{\gamma_{3} \alpha_{2}}{\gamma_{2} \alpha_{3}}=\cdots=\frac{\gamma_{m_{2}-m_{1}+1} \alpha_{m_{2}-m_{1}}}{\gamma_{m_{2}-m_{1}} \alpha_{m_{2}-m_{1}+1}}
$$

Again, from equations (2), we find that

$$
b_{3}=\frac{\gamma_{1}}{\alpha_{1}} d_{3}=\frac{\gamma_{2}}{\alpha_{2}} d_{2}, \text { whence } \frac{d_{3}}{d_{2}}=\frac{\gamma_{2} \alpha_{1}}{\gamma_{1} \alpha_{2}}=s .
$$

By a repetition of such an argument, we can show that

Hence

$$
s=\frac{d_{2}}{d_{1}}=\frac{d_{3}}{d_{2}}=\cdots=\frac{d_{m_{1}}}{d_{m_{1}-1}} .
$$

$$
D=d_{1}\left[1, s, s^{2}, \cdots, s^{m_{1}-1}\right] \text {. }
$$

Equating the last elements in each of the matrix equations (2), we find that by equations (3), since $d_{m_{1}}=d_{1} s^{m_{1}-1}$,

$$
\begin{aligned}
& b_{m_{1}+1}=\frac{\gamma_{2}}{\alpha_{2}} d_{m_{1}}=\frac{\gamma_{2} \alpha_{1}}{\gamma_{1} \alpha_{2}} \frac{\gamma_{1}}{\alpha_{1}} d_{1} s^{m_{1}-1}=\frac{\gamma_{1}}{\alpha_{1}} d_{1} s^{m_{1}}=\frac{c_{1}}{a_{1}} d_{1} s^{m_{1}}, \\
& b_{m_{1}+2}=\frac{\gamma_{3}}{\alpha_{3}} d_{m_{1}}=\frac{\gamma_{3} \alpha_{2}}{\gamma_{2} \alpha_{3}} \frac{\gamma_{2} \alpha_{1}}{\gamma_{1} \alpha_{2}} \frac{\gamma_{1}}{\alpha_{1}} d_{1} s^{m_{1}-1}=\frac{\gamma_{1}}{\alpha_{1}} d_{1} s^{m_{1}+1}=\frac{c_{1}}{a_{1}} d_{1} s^{m_{1}+1}, \\
& b_{m_{2}}=\frac{\gamma_{m_{2}-m_{1}+1}}{\alpha_{m_{2}-m_{1}+1}} d_{m_{1}}=\cdots=\frac{\gamma_{1}}{\alpha_{1}} d_{1} s^{m_{2}-1}=\frac{c_{1}}{a_{1}} d_{1} s^{m_{2}-1} .
\end{aligned}
$$

Hence, since

$$
\left[b_{1}, \cdots, b_{m_{1}}\right]=\frac{\gamma_{1}}{\alpha_{1}} d_{1}\left[1, s, \cdots, s^{m_{1}-1}\right]=\frac{c_{1}}{a_{1}} d_{1}\left[1, s, \cdots, s^{m_{1}-1}\right],
$$

we may write

$B=\left[b_{1}, \cdots, b_{m_{2}}\right]=\frac{c_{1}}{a_{1}} d_{1}\left[1, s, \cdots, s^{m_{2}-1}\right]=b_{1}\left[1, s, \cdots, s^{m_{2}-1}\right]$.

We have now shown that with the possible exceptions of the elements $a_{2} b_{1}, a_{3} b_{1}, \cdots, a_{m_{1}} b_{1}$, every element of $A\langle B\rangle$ is $s$ times the preceding element; that is, every element, with the possible exception of the

(4) $\quad\left(m_{2}+1\right)$ th, $\left(2 m_{2}+1\right)$ th, $\cdots,\left(m_{2}\left(m_{1}-1\right)+1\right)$ th 
elements. But this is also true of $C\langle D\rangle$ with the possible exception of the

$$
\left(m_{1}+1\right) \text { th, }\left(2 m_{1}+1\right) \text { th, } \cdots,\left(m_{1}\left(m_{2}-1\right)+1\right) \text { th }
$$

elements. But, since $\left(m_{1}, m_{2}\right)=1$, no member of the set (4) is a member of the set (5); and since the elements of $A\langle B\rangle$ are identical with those of $C\langle D\rangle$, there are no exceptions and every element of $A\langle B\rangle$ or $C\langle D\rangle$ is $s$ times the preceding element.

Hence

$$
a_{3} b_{1}=s a_{1} b_{m_{2}}=s a_{1} b_{1} s^{m_{2}-1},
$$

so that

$$
a_{2}=a_{1} s^{m_{2}}
$$

and similarly

$$
\begin{gathered}
a_{3}=a_{2} s^{m_{2}}=a_{1} s^{2 m_{2}} \\
\cdot \cdot \cdot \cdot \cdot \\
a_{m_{1}}=a_{1} s^{m_{2}\left(m_{1}-1\right)} .
\end{gathered}
$$

It follows that

$$
A=a_{1}\left[1, s^{m_{2}}, s^{2 m_{2}}, \cdots, s^{m_{2}\left(m_{1}-1\right)}\right],
$$

and similarly

$$
C=c_{1}\left[1, s^{m_{1}}, s^{2 m_{1}}, \cdots, s^{m_{1}\left(m_{2}-1\right)}\right] .
$$

We have not yet considered all the $m_{1} m_{2}$ equations connecting the elements of $A, B, C, D$; but, since the above values of $A, B, C, D$ give a solution for any values of the arbitrary quantities $a_{1}, c_{1}, d_{1}, s$, they also give the most general solution.

3. A More General Case. We shall now consider a more general case, where $A$ and $D$ are rectangular matrices of $n_{1}$ rows and $m_{1}$ columns, where $B$ and $C$ are rectangular matrices of $n_{2}$ rows and $m_{2}$ columns, and where $\left(n_{1}, n_{2}\right)=1$, and $\left(m_{1}, m_{2}\right)=1$. Let

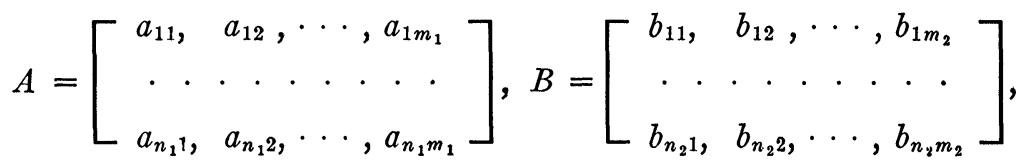

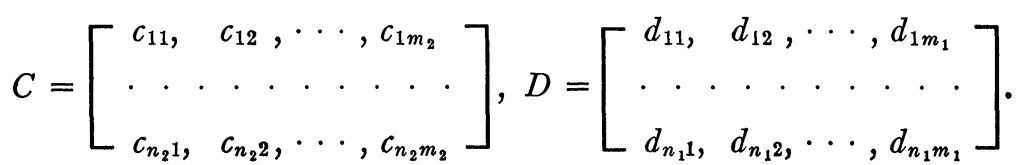


By equating the first rows of $A\langle B\rangle$ and $C\langle D\rangle$ we obtain from $\S 2$ the relation

$$
\left[b_{11}, b_{12}, \cdots, b_{1 m_{2}}\right]=b_{11}\left[1, s, \cdots, s^{m_{2}-1}\right] .
$$

Similarly, by equating the second rows of $A\langle B\rangle$ and $C\langle D\rangle$ we deduce that

$$
\left[b_{21}, b_{22}, \cdots, b_{2 m_{2}}\right]=b_{21}\left[1, s, \cdots, s^{m_{2}-1}\right],
$$

for the $a$ 's and $c$ 's occurring are the same in both rows and hence the $s$ must be the same in (7) as in (6). Proceeding in this way with the rows of $A\langle B\rangle$ and $C\langle D\rangle$, we obtain eventually

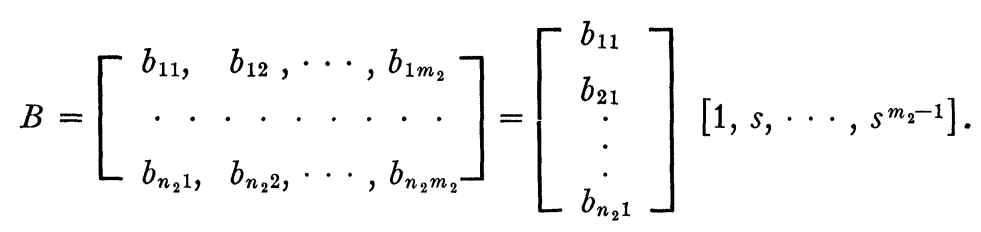

We shall find it more convenient to denote the first factor on the right hand side by $\left\{b_{11}, b_{21}, \cdots, b_{n_{2}}\right\}$, as is frequently done, that is, the curly brackets denote a column vector.

Now, by equating the first columns in $A\langle B\rangle$ and $C\langle D\rangle$, we obtain, in the same manner as (6) was obtained, the relation

$$
\left\{b_{11}, b_{21}, \cdots, b_{n_{2} 1}\right\}=b_{11}\left\{1, t, t^{2}, \cdots, t^{n_{2}-1}\right\},
$$

where $t$ is a new arbitrary quantity. Hence

$$
B=b_{11}\left\{1, t, \cdots, t^{n_{2}-1}\right\}\left[1, s, \cdots, s^{m_{2}-1}\right],
$$

and in the same manner

$$
\begin{gathered}
D=d_{11}\left\{1, t, \cdots, t^{n_{1}-1}\right\}\left[1, s, \cdots, s^{m_{1}-1}\right], \\
A=a_{11}\left\{1, t^{n_{2}}, t^{2 n_{2}}, \cdots, t^{n_{2}\left(n_{1}-1\right)}\right\}\left[1, s^{m_{2}}, s^{2 m_{2}}, \cdots, s^{m_{2}\left(m_{1}-1\right)}\right], \\
C=c_{11}\left\{1, t^{n_{1}}, t^{2 n_{1}}, \cdots, t^{n_{1}\left(n_{2}-1\right)}\right\}\left[1, s^{m_{1}}, s^{2 m_{1}}, \cdots, s^{m_{1}\left(m_{2}-1\right)}\right],
\end{gathered}
$$

where $a_{11} b_{11}=c_{11} d_{11}$. It follows that since the above values of $A, B, C, D$ give a solution of $A\langle B\rangle=C\langle D\rangle$, for any values of $a_{11}, c_{11}, d_{11}, s, t$, they give the most general solution.

4. The Most General Case. We shall now consider the most general case and show that its solution is dependent upon the one just obtained. Suppose that the matrices $A, B, C, D$ have 
$n_{1}, n_{2}, n_{3}, n_{4}$ rows and $m_{1}, m_{2}, m_{3}, m_{4}$ columns respectively. Since $A\langle B\rangle=C\langle D\rangle$,

$$
n_{1} n_{2}=n_{3} n_{4}, \text { and } m_{1} m_{2}=m_{3} m_{4} .
$$

Let the highest common factor of $n_{1}$ and $n_{3}$ be $k_{1}$. We write this $\left(n_{1}, n_{3}\right)=k_{1}$. Let $n_{1}=\nu_{1} k_{1}$ and $n_{3}=\nu_{3} k_{1}$, where $\left(\nu_{1}, \nu_{3}\right)=1$. Similarly, let $n_{2}=\nu_{2} k_{2}$ and $n_{4}=\nu_{4} k_{2}$, where $\left(\nu_{2}, \nu_{4}\right)=1$; let $m_{1}=\mu_{1} h_{1}$ and $m_{3}=\mu_{3} h_{1}$, where $\left(\mu_{1}, \mu_{3}\right)=1$; and let $m_{2}=\mu_{2} h_{2}$ and $m_{4}=\mu_{4} h_{2}$, where $\left(\mu_{2}, \mu_{4}\right)=1$. From equation (8) $\nu_{1} \nu_{2} k_{1} k_{2}=\nu_{3} \nu_{4} k_{1} k_{2}$ and therefore $\nu_{1} \nu_{2}=\nu_{3} \nu_{4}$. Now since $\left(\nu_{1}, \nu_{3}\right)=1, \nu_{1}$ must be a factor of $\nu_{4}$, and since $\left(\nu_{2}, \nu_{4}\right)=1, \nu_{4}$ must be a factor of $\nu_{1}$. Hence $\nu_{1}=\nu_{4}$ and $\nu_{2}=\nu_{3}$, also $\left(\nu_{1}, \nu_{2}\right)=1$. Similarly $\mu_{1}=\mu_{4}$ and $\mu_{2}=\mu_{3}$, also $\left(\mu_{1}, \mu_{2}\right)=1$. The procedure is now quite simple, although it is somewhat difficult to explain in writing. Consider the very simple case

$$
\begin{aligned}
& {\left[a_{1}, a_{2}, a_{3}, a_{4}, a_{5}, a_{6}\right]\left\langle\left[b_{1}, b_{2}, b_{3}, b_{4}\right]\right\rangle} \\
& =\left[c_{1}, c_{2}, c_{3}, c_{4}\right]\left\langle\left[d_{1}, d_{2}, d_{3}, d_{4}, d_{5}, d_{6}\right]\right\rangle .
\end{aligned}
$$

In this example, $h_{1}=2$, and we see that the above equation can be split up into the two equations

$$
\begin{aligned}
& {\left[a_{1}, a_{2}, a_{3}\right]\left\langle\left[b_{1}, b_{2}, b_{3}, b_{4}\right]\right\rangle=\left[c_{1}, c_{2}\right]\left\langle\left[d_{1}, d_{2}, d_{3}, d_{4}, d_{5}, d_{6}\right]\right\rangle,} \\
& {\left[a_{4}, a_{5}, a_{6}\right]\left\langle\left[b_{1}, b_{2}, b_{3}, b_{4}\right]\right\rangle=\left[c_{3}, c_{4}\right]\left\langle\left[d_{1}, d_{2}, d_{3}, d_{4}, d_{5}, d_{6}\right]\right\rangle .}
\end{aligned}
$$

In this example $h_{2}=2$, and we can split up each of the above into two equations and so we can reduce this case to the following four examples of the case considered in $\$ 2$ :

$$
\begin{aligned}
& {\left[a_{1}, a_{2}, a_{3}\right]\left\langle\left[b_{1}, b_{3}\right]\right\rangle=\left[c_{1}, c_{2}\right]\left\langle\left[d_{1}, d_{3}, d_{5}\right]\right\rangle,} \\
& {\left[a_{1}, a_{2}, a_{3}\right]\left\langle\left[b_{2}, b_{4}\right]\right\rangle=\left[c_{1}, c_{2}\right]\left\langle\left[d_{2}, d_{4}, d_{6}\right]\right\rangle,} \\
& {\left[a_{4}, a_{5}, a_{6}\right]\left\langle\left[b_{1}, b_{3}\right]\right\rangle=\left[c_{3}, c_{4}\right]\left\langle\left[d_{1}, d_{3}, d_{5}\right]\right\rangle,} \\
& {\left[a_{4}, a_{5}, a_{6}\right]\left\langle\left[b_{2}, b_{4}\right]\right\rangle=\left[c_{3}, c_{4}\right]\left\langle\left[d_{2}, d_{4}, d_{6}\right]\right\rangle .}
\end{aligned}
$$

In the most general case, we can split up the equation

$$
A\langle B\rangle=C\langle D\rangle
$$

into $k_{1} k_{2} h_{1} h_{2}$ equations

$$
A_{x y}\left\langle B_{z u}\right\rangle=C_{x y}\left\langle D_{z u}\right\rangle,
$$

where $x=1,2, \cdots, k_{1} ; y=1,2, \cdots, h_{1} ; z=1,2, \cdots, k_{2}$; $u=1,2, \cdots, h_{2}$, and where $A_{x y}$ is the matrix of $\nu_{1}$ rows and $\mu_{1}$ 
columns whose $i j$ th element is $a_{(x-1) \nu_{1}+i,(y-1) \mu_{1}+j}$. That is to say, $A_{x y}=\left[a_{(x-1) \nu_{1}+i,(y-1) \mu_{1}+j}\right]$ has $\nu_{1}$ rows and $\mu_{1}$ columns. Similarly, $C_{x y}=\left[c_{(x-1) \nu_{2}+i,(y-1) \mu_{2}+j}\right]$ has $\nu_{2}$ rows and $\mu_{2}$ columns; while $B_{z u}=\left[b_{(i-1) k_{2}+z,(j-1) h_{2}+u}\right]$ has $\nu_{2}$ rows and $\mu_{2}$ columns and $D_{z u}=\left[d_{(i-1) k_{2}+z,(j-1) h_{2}+u}\right]$ has $\nu_{1}$ rows and $\mu_{1}$ columns. But, since $\left(\nu_{1}, \nu_{2}\right)=1$ and $\left(\mu_{1}, \mu_{2}\right)=1$, the most general case is composed of $k_{1} k_{2} h_{1} h_{2}$ examples of the case treated in $\S 3$. For brevity, let us write

$$
\begin{aligned}
E & =\left\{1, t^{\nu_{2}}, \cdots, t^{\nu_{2}\left(\nu_{1}-1\right)}\right\}\left[1, s^{\mu_{2}}, \cdots, s^{\mu_{2}\left(\mu_{1}-1\right)}\right] \\
F & =\left\{1, t, \cdots, t^{\nu_{2}-1}\right\}\left[1, s, \cdots, s^{\mu_{2}-1}\right], \\
G & =\left\{1, t^{\nu_{1}}, \cdots, t^{\nu_{1}\left(\nu_{2}-1\right)}\right\}\left[1, s^{\mu_{1}}, \cdots, s^{\mu_{1}\left(\mu_{2}-1\right)}\right], \\
H & =\left\{1, t, \cdots, t^{\nu_{1}-1}\right\}\left[1, s, \cdots, s^{\mu_{1}-1}\right] .
\end{aligned}
$$

Now, solving $A_{11}\left\langle B_{11}\right\rangle=C_{11}\left\langle D_{11}\right\rangle$ by the method of $\$ 3$, we find

$$
A_{11}=a_{11} E, \quad B_{11}=b_{11} F, \quad C_{11}=c_{11} G, \quad D_{11}=d_{11} H,
$$

where $a_{11} b_{11}=c_{11} d_{11}$. Similarly solving $A_{x y}\left\langle B_{z u}\right\rangle=C_{x y}\left\langle D_{z u}\right\rangle$, we have

$$
\begin{array}{ll}
A_{x y}=a_{(x-1) \nu_{1}+1,(y-1) \mu_{1}+1} E, & B_{z u}=b_{z u} F, \\
C_{x y}=a_{(x-1) \nu_{2}+1,(y-1) \mu_{2}+1} G, & D_{z u}=d_{z u} H,
\end{array}
$$

where $a_{(x-1) \nu_{1}+1,(y-1) \mu_{1}+1} b_{z u}=c_{(x-1) \nu_{2}+1,(y-1) \mu_{2}+1} d_{z u}$ for all values of $x, y, z, u$. Hence

$$
\frac{a_{(x-1) \nu_{1}+1,(y-1) \mu_{1}+1}}{c_{(x-1) \nu_{2}+1,(y-1) \mu_{2}+1}}=\frac{d_{z u}}{b_{z u}}=q,
$$

where $q$ is a constant for all values of $x, y, z, u$. We notice that $E, F, G, H$ are the same for all values of $x, y, z, u$, for otherwise we would find some $B_{z u}$ having two different values at once. It follows that

$$
\begin{aligned}
& A=\left[\begin{array}{c}
A_{11}, \cdots, A_{1 h_{1}} \\
\cdot \cdot \cdot \cdot \cdot \cdot \cdot] \\
A_{k_{1} 1}, \cdots, A_{k_{1} h_{1}}
\end{array}\right]
\end{aligned}
$$

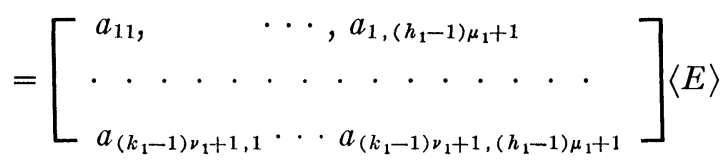




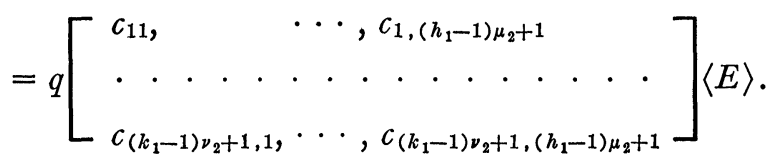

Similarly

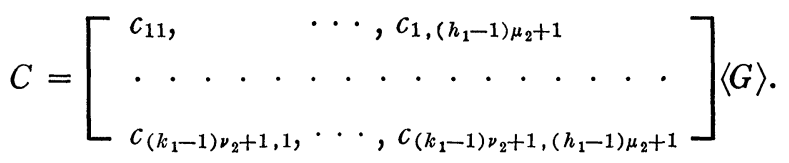

In the same way it can be shown that

$$
B=F\left\langle\left[\begin{array}{c}
b_{11}, \cdots, b_{1 h_{2}} \\
\cdot . . . \\
b_{k_{2} 1}, \cdots, b_{k_{2} h_{2}}
\end{array}\right]\right\rangle \text { and } D=q H\left\langle\left[\begin{array}{c}
b_{11}, \cdots, b_{1 h_{2}} \\
\cdot . . . \\
b_{k_{2} 1}, \cdots, b_{k_{2} h_{2}}
\end{array}\right]\right\rangle \text {. }
$$

The above values for $A, B, C, D$ give the most general solution of the equation

$$
A\langle B\rangle=C\langle D\rangle \text {. }
$$

The University of Edinburgh 